\title{
A large data resource of genomic copy number variation across neurodevelopmental disorders
}

Mehdi Zarrei $^{1,2}$, Christie L. Burton ${ }^{3}$, Worrawat Engchuan ${ }^{1,2}$, Edwin J. Young (iD ${ }^{4}$, Edward J. Higginbotham (iD ${ }^{1,2,5}$, Jeffrey R. MacDonald (iD) Brett Trost iD $^{1,2}$, Ada J. S. Chan ${ }^{1,2,5}$, Susan Walker ${ }^{1}$, Sylvia Lamoureux ${ }^{1}$, Tracy Heung ${ }^{6}$, Bahareh A. Mojarad ${ }^{2}$, Barbara Kellam ${ }^{1}$, Tara Paton ${ }^{1}$, Muhammad Faheem ${ }^{1,2}$, Karin Miron ${ }^{1,2}$, Chao Lu ${ }^{1}$, Ting Wang ${ }^{1}$, Kozue Samler ${ }^{1}$, Xiaolin Wang ${ }^{1}$, Gregory Costain ${ }^{7,8}$, Ny Hoang ${ }^{2,5,9}$, Giovanna Pellecchia (1D) ', John Wei ${ }^{1}$, Rohan V. Patel (1D) ${ }^{1}$, Bhooma Thiruvahindrapuram ${ }^{1}$, Maian Roifman ${ }^{7,10,11}$, Daniele Merico ${ }^{1,12}$, Tara Goodale ${ }^{3}$, Irene Drmic ${ }^{13}$, Marsha Speevak ${ }^{14}$, Jennifer L. Howe ${ }^{1}$, Ryan K. C. Yuen (iD ${ }^{1,2}$, Janet A. Buchanan ${ }^{1}$, Jacob A. S. Vorstman ${ }^{15,16}$, Christian R. Marshall (iD ${ }^{1,4,17}$, Richard F. Wintle (iD) ${ }^{1}$, David R. Rosenberg ${ }^{18,19}$, Gregory L. Hanna ${ }^{20}$, Marc Woodbury-Smith ${ }^{1,21}$, Cheryl Cytrynbaum (iD ${ }^{2,5,7,22}$, Lonnie Zwaigenbaum ${ }^{23}$, Mayada Elsabbagh ${ }^{24}$, Janine Flanagan ${ }^{11}$, Bridget A. Fernandez ${ }^{25}$ Melissa T. Carter ${ }^{26}$, Peter Szatmari ${ }^{15,27,28}$, Wendy Roberts ${ }^{16}$, Jason Lerch ${ }^{29,30}$, Xudong Liu ${ }^{31}$, Rob Nicolson (iD ${ }^{32,33}$, Stelios Georgiades ${ }^{34}$, Rosanna Weksberg ${ }^{2,7,5}$, Paul D. Arnold ${ }^{2,35,36}$, Anne S. Bassett ${ }^{6,15,37}$, Jennifer Crosbie ${ }^{3,15}$, Russell Schachar ${ }^{3,15,38}$, Dimitri J. Stavropoulos ${ }^{4}$, Evdokia Anagnostou ${ }^{39}$ and Stephen W. Scherer $\mathbb{D}^{1,2,5,40 *}$

Copy number variations (CNVs) are implicated across many neurodevelopmental disorders (NDDs) and contribute to their shared genetic etiology. Multiple studies have attempted to identify shared etiology among NDDs, but this is the first genome-wide CNV analysis across autism spectrum disorder (ASD), attention deficit hyperactivity disorder (ADHD), schizophrenia (SCZ), and obsessivecompulsive disorder (OCD) at once. Using microarray (Affymetrix CytoScan HD), we genotyped 2,691 subjects diagnosed with an NDD (204 SCZ, 1,838 ASD, 427 ADHD and 222 OCD) and 1,769 family members, mainly parents. We identified rare CNVs, defined as those found in $<0.1 \%$ of 10,851 population control samples. We found clinically relevant CNVs (broadly defined) in 284 (10.5\%) of total subjects, including 22 (10.8\%) among subjects with SCZ, 209 (11.4\%) with ASD, 40 (9.4\%) with ADHD, and 13 (5.6\%) with OCD. Among all NDD subjects, we identified $17(0.63 \%)$ with aneuploidies and 115 (4.3\%) with known genomic disorder variants. We searched further for genes impacted by different CNVs in multiple disorders. Examples of NDD-associated genes linked across more than one disorder (listed in order of occurrence frequency) are NRXN1, SEH1L, LDLRAD4, GNAL, GNG13, MKRN1, DCTN2, KNDC1, PCMTD2, KIF5A, SYNM, and long non-coding RNAs: AK127244 and PTCHD1-AS. We demonstrated that CNVs impacting the same genes could potentially contribute to the etiology of multiple NDDs. The CNVs identified will serve as a useful resource for both research and diagnostic laboratories for prioritization of variants.

npj Genomic Medicine (2019)4:26

; https://doi.org/10.1038/s41525-019-0098-3

\section{INTRODUCTION}

Genomic copy number variations (CNVs) are structural variations that involve deletions and/or duplications of segments of DNA. Their impact is not necessarily harmful, but loss, increase, or disruption of genes is often associated with, and can underlie human disease, including neurodevelopmental disorders (NDDs). ${ }^{1-6}$ Rare CNVs have been extensively studied in autism spectrum disorder (ASD), ${ }^{7-9}$ attention deficit hyperactivity disorder (ADHD), ${ }^{10}$ schizophrenia (SCZ), ${ }^{11,12}$ and less so, obsessive compulsive disorder (OCD). ${ }^{13,14}$ Various NDDs share genomic structural

\footnotetext{
${ }^{1}$ The Centre for Applied Genomics, The Hospital for Sick Children, Toronto, ON, Canada. ${ }^{2}$ Program in Genetics and Genome Biology, The Hospital for Sick Children, Toronto, ON, Canada. ${ }^{3}$ Neurosciences and Mental Health Program, The Hospital for Sick Children, Toronto, ON, Canada. ${ }^{4}$ Genome Diagnostics, Department of Paediatric Laboratory Medicine, The Hospital for Sick Children, Toronto, ON, Canada. ${ }^{5}$ Department of Molecular Genetics, University of Toronto, Toronto, ON, Canada. ${ }^{6}$ Clinical Genetics Research Program, Centre for Addiction and Mental Health, Toronto, ON, Canada. ${ }^{7}$ Division of Clinical and Metabolic Genetics, The Hospital for Sick Children, Toronto, ON, Canada. ${ }^{8}$ Medical Genetics Residency Training Program, University of Toronto, Toronto, ON, Canada. ${ }^{9}$ Department of Genetic Counselling, The Hospital for Sick Children, Toronto, ON, Canada. ${ }^{10}$ The Prenatal Diagnosis and Medical Genetics Program, Department of Obstetrics and Gynecology, Mount Sinai Hospital, Toronto, ON, Canada. ${ }^{11}$ Department of Paediatrics, University of Toronto, Toronto, ON, Canada. ${ }^{12}$ Deep Genomics Inc., Toronto, ON, Canada. ${ }^{13}$ Hamilton Health Sciences, Ron Joyce Children's Health Centre, Hamilton, On, Canada. ${ }^{14}$ Trillium Health Partners Credit Valley Site, Mississauga, Ontario, Canada. ${ }^{15}$ Department of Psychiatry, University of Toronto, Toronto, ON, Canada. ${ }^{16}$ Autism Research Unit, The Hospital for Sick Children, Toronto, ON, Canada. ${ }^{17}$ Laboratory Medicine and Pathobiology, University of Toronto, Toronto, ON, Canada. ${ }^{18}$ Department of Psychiatry and Behavioral Neurosciences, Wayne State University, Detroit, MI, USA. ${ }^{19}$ The Children's Hospital of Michigan, Detroit, MI, United States. ${ }^{20}$ Department of Psychiatry, University of Michigan, Ann Arbor, MI, USA. ${ }^{21}$ Institute of Neuroscience, Newcastle University, Newcastle upon Tyne, UK. ${ }^{22}$ Dalla Lana School of Public Health and the Department of Family and Community Medicine, University of Toronto, Toronto, ON, Canada. ${ }^{23}$ Department of Pediatrics, University of Alberta, Edmonton, AB, Canada. ${ }^{24}$ Montreal Neurological Institute, McGill University, Montreal, QC, Canada. ${ }^{25}$ Discipline of Genetics, Faculty of Medicine, Memorial University of Newfoundland, St. John's, NL, Canada. ${ }^{26}$ Regional Genetics Program, The Children's Hospital of Eastern Ontario, Ottawa, ON, Canada. ${ }^{27}$ Centre for Addiction and Mental Health, Toronto, ON, Canada. ${ }^{28}$ Department of Psychiatry, The Hospital for Sick Children, Toronto, ON, Canada. ${ }^{29}$ Mouse Imaging Centre, Hospital for Sick Children, Toronto, ON, Canada. ${ }^{30}$ Department of Medical Biophysics, The University of Toronto, Toronto, ON, Canada. ${ }^{31}$ Department of Psychiatry, Queen's University, Kinston, ON, Canada. ${ }^{32}$ Children's Health Research Institute, London, ON, Canada. ${ }^{33}$ Western University, London, ON, Canada. ${ }^{34}$ Department of Psychiatry and Behavioural Neurosciences, McMaster University, Hamilton, ON, Canada. ${ }^{35}$ Mathison Centre for Mental Health Research and Education, University of Calgary, Calgary, AB, Canada. ${ }^{36}$ Departments of Psychiatry and Medical Genetics, Cumming School of Medicine, University of Calgary, Calgary, AB, Canada. ${ }^{37}$ The Dalglish Family 22q Clinic, Toronto General Hospital, Toronto, ON, Canada. ${ }^{38}$ Institute of Medical Science, University of Toronto, Toronto, ON, Canada. ${ }^{39} \mathrm{Holland}$ Bloorview Kids Rehabilitation Hospital, University of Toronto, Toronto, ON, Canada. ${ }^{40}$ Department of Molecular Genetics and McLaughlin Centre, University of Toronto, Toronto, ON, Canada. *email: stephen.scherer@sickkids.ca
} 
M. Zarrei et al.

Table 1. Stratification of 4,460 samples in the cross-disorder CNV analysis

\begin{tabular}{|c|c|c|c|c|c|c|c|c|c|}
\hline Disorder & Samples $^{1}$ & Cases $^{2}$ & Adult $^{3}$ & Sex ratio (male/female) & Only probands ${ }^{4}$ & Only one parent ${ }^{5}$ & Trios $^{6}$ & Quartets $^{7}$ & Others $^{8}$ \\
\hline ADHD & 427 & 427 & 0 & $3.7(337 / 90)$ & 427 & 0 & 0 & 0 & 0 \\
\hline OCD & 564 & 222 & 0 & $0.9(107 / 115)$ & 49 & 6 & 167 & 0 & 0 \\
\hline All & 4,460 & 2,691 & $10.3 \%$ & $3.2(2,055 / 638)$ & 1,769 & 131 & 485 & 126 & 17 \\
\hline
\end{tabular}

$A S D$ autism spectrum disorder, $A D H D$ attention deficit hyperactivity disorder, $S C Z$ schizophrenia, OCD obsessive compulsive disorder

${ }^{1}$ Count of all samples that passed our stringent QC, including assessing genotype quality, removing duplicated samples or those with non-mendelian segregation implying alternative parental relationships. This includes parents, extended family members, and siblings. ${ }^{2}$ Includes probands and affected sibling (s). Affected parents are not considered. ${ }^{3}$ Proportion of cases at least 18 years of age at the time of diagnosis (for schizophrenia) or time of DNA submission for the microarray genotyping (for ASD $(n=73)$ ). ${ }^{4}$ Includes affected individuals for whom parents were not sampled or failed QC. No parents were sampled for ADHD. ${ }^{5}$ Affected individuals with one parent sampled or passed QC. ${ }^{6}$ Proband and both parents. ${ }^{7}$ Proband, one affected sibling and both parents. ${ }^{8}$ Families with three or more affected individuals (15 families with three, one family with four, and one with five affected children) for ASD. ${ }^{9}$ Includes 231 samples of extended family members or parents, including affected individuals. ${ }^{10}$ Of SCZ cases, 31 had intellectual disability

variations, including CNVs that perturb the same genes. ${ }^{1,15}$ For example, deletions or duplications affecting coding sequences of NRXN1 or CNTN6 have been implicated in ASD, ADHD, and SCZ. ${ }^{16,17}$

A shared genomic etiology across multiple disorders is supported through genome-wide association studies ${ }^{18}$ or analyzing small loss-of-function (LoF) variations. ${ }^{19}$ However, CNV analysis across disorders has been limited; e.g., ADHD and ASD, ${ }^{10}$ ASD and $\mathrm{SCZ}_{1}^{20}$ reviews on selected well-established genomic disorders such as $16 \mathrm{p} 11.2$ deletions and duplications, ${ }^{21}$ or a meta-analysis of CNVs on a single gene $\left(N R X N 1^{16}\right)$. To date, there has been no genome-wide study of rare CNVs identified using an identical technology, encompassing ASD, ADHD, OCD, and SCZ. An identical method for interrogating CNVs across multiple disorders increases the chance of finding rare CNVs with cross-disorder implications. These could be missed if multiple technologies with different detection sensitivities were applied.

This project was established to provide a public resource of CNV samples with NDDs mostly from the province of Ontario, Canada, all genotyped on same microarray platform: the Affymetrix CytoScan HD platform, which consists of 1.9 million copy number markers and 750,000 genotype-able single nucleotides polymorphisms. A CNV resource of control population samples was published earlier under the Ontario Population Genomics Platform. $^{22}$

In creating this data resource, we aimed to: (i) catalogue CNVs that are clinically relevant to each of ASD, ADHD, OCD, and SCZ, and (ii) identify genes and loci with CNVs that are shared among different NDDs. Where available, we analyzed whole genome (WGS) or whole exome sequence (WES) data, in search of variants that were not detected by microarray. The relevant genotypes and CNVs are available in dbGaP (accession number phs001881.v1.p1) and dbVar (accession number nstd173), respectively.

\section{RESULTS}

Sample description and detection of CNVs

We analyzed 4,460 samples ascertained for four NDDs; 2,691 $(60.3 \%)$ were from individuals recruited because of the diagnosis of one of these disorders, and the rest from apparently unaffected family members (Table 1, Supplementary Table 1A). ASD, ADHD, OCD, and SCZ cases were ascertained using criteria explained previously (Supplementary Information). 8,10,13,23,24 Childhood onset OCD is not classically considered an NDD in the International Classification of Disease (ICD-11) or Diagnostic and Statistical Manual of Mental Disorders (DSM-5), but in view of its early onset, male preponderance and association with imaging findings, we included OCD in this study as others often do. Similarly, since SCZ has both neural and genetic correlates, including some evidence of overlap or sharing genetic risk with NDDs, we considered SCZ as well. The majority of samples (68.1\%) were ascertained for ASD, with the others distributed approximately equally among the other disorders. The male:female sex ratio was almost 4:1 for ASD and ADHD, 2:1 for SCZ, and 1:1 in the pediatric $O C D$ cases (Table 1). Different family structures were sampled in the four sub-groups: some ASD and SCZ families included multiple affected individuals; some OCD samples were in trios (i.e., affected proband and both parents; details in Table 1, Supplementary Table 1A). We did not genotype parents of cases for ADHD (Table 1, Supplementary Table 1A). We defined a high confidence set of CNVs (Supplementary Table 1B) as those identified using two different detection algorithms, as previously described. ${ }^{25}$ Rare variants were those with a frequency of less than $0.1 \%$ in a 10,851 subject population control sample genotyped in multiple microarray platforms, including the Affymetrix CytoScan HD. ${ }^{25}$ We also analyzed prioritized CNVs from our previous publications on $\mathrm{ADHD}^{10}$ and $\mathrm{SCZ} .{ }^{11,24}$ We had information with respect to intellectual disability (ID) for some cases. ID including borderline intellect and non-verbal learning disability was comorbid with SCZ in 31/204 cases (16.9\%), ${ }^{11}$ with ASD in 149/ 599 cases (24.9\%), and with ADHD in $3 / 427$ cases (0.7\%). No OCD case had ID.

\section{Clinically relevant CNVs}

Clinically relevant CNVs included five categories: aneuploidies, large CNVs ( $>3 \mathrm{Mb}$ ), CNVs consistent with known recurrent genomic disorders, those impacting genes previously established to be associated with NDDs, and all de novo CNVs (i.e., not found in either parent) (details in Table 2). We found 306 clinically relevant CNVs in 284 of 2,691 NDD cases (10.5\%) (Tables 2, 3, Supplementary Table 1C). Of these CNVs, 115 found in 111/2,691 cases $(4.3 \%)$ were "clinically significant" or "likely clinically significant" variants, as evaluated by expert clinical geneticists according to American College of Medical Genetics and Genomics guidelines. ${ }^{26}$ We did not find evidence for uniparental disomy. The clinically relevant autosomal deletions, and chromosome $\mathrm{X}$ deletions in females, were all single copy.

The first category included aneuploidies: trisomy 21, 47, XXY, 47, $X Y Y$, and $45, X$, found in $17 / 2,691$ cases ( $0.63 \%)$, only among ADHD or ASD cases (category A in Tables 2, 3; Fig. 1). The second CNV category contained variants larger than $3 \mathrm{Mb}$, but excluding those 
Table 2. Summary of cases carrying a CNV deemed relevant to neurodevelopmental disorders CNVs stratified by the disorder and variant type

\begin{tabular}{|c|c|c|c|c|c|}
\hline Category & ASD (\%) & ADHD (\%) & OCD (\%) & SCZ (\%) & All cases $(\%)$ \\
\hline B: large CNVs $(>3 \mathrm{Mb})^{1}$ & $16(0.9)$ & $4(0.9)$ & 0 & $3(1.5)$ & $23(0.85)$ \\
\hline D: de novo ${ }^{3}$ & $31(6.9)$ & $N A^{5}$ & $3(1.8)$ & $1(\mathrm{NA})^{6}$ & $35(5.6)$ \\
\hline E: others ${ }^{4}$ & $89(4.9)$ & $14(3.3)$ & $8(3.6)$ & $6(2.9)$ & $117(4.4)$ \\
\hline Sum of unique samples across all categories ${ }^{7}$ & $209(11.4)$ & $40(9.4)$ & $13(5.6)$ & $22(10.8)$ & $284(10.5)^{8}$ \\
\hline
\end{tabular}

${ }^{1}$ This category included variants larger than $3 \mathrm{Mb}$ but not aneuploidies or variants of known recurrent genomic disorder loci larger than $3 \mathrm{Mb}$, i.e., $15 \mathrm{q} 11-13$ duplication (Table 3, Supplementary Table 1C). ${ }^{2}$ Prevalence of known recurrent genomic syndromes in the general population is $0.8-1.0 \%$. ${ }^{3}$ This category includes de novo variants from aneuploidies, large CNVs and genomic disorder loci. The rate of de novo CNVs in the general population is $0.9-1.4 \%{ }^{43}$. ${ }^{4}$ This category included all other NDD relevant CNVs. ${ }^{5}$ Indicates that we did not sample parents of ADHD cases to establish inheritance pattern of variants. ${ }^{6}$ We sampled only 13 trios; most SCZ samples were unrelated. Sum of the counts in each column is higher in some cases than the total number of cases with relevant CNVs in the corresponding column, due to the fact that (i) some subjects might carry multiple relevant variants, and (ii) de novo category includes CNVs from aneuploidy, large CNVs, and the genomic disorder loci. ${ }^{7}$ Numbers of prioritized CNVs for ASD, ADHD, OCD, and SCZ were 223, 43, 17, and 23, respectively. ${ }^{8}$ Total number of prioritized CNVs across all cases was 306 . The coordinates for clinically relevant CNVs are in Supplementary Table $1 \mathrm{C}$

associated with recurrent genomic disorders and aneuploidies (category B in Tables 2, 3). We found these large variants in 23/ 2,691 NDD cases $(0.85 \%)$, with none among the OCD cases. The third category was the variants associated with known recurrent genomic disorders, found in $115 / 2,691$ cases (4.3\%; category $C$ in Tables 2, 3; Fig. 1). The most frequent were 16p13.11 duplications (17 cases), 15q11.2 deletions (breakpoint (BP1-BP2) (16 cases), and $15 q 11.2$ duplications (BP1-BP2) (15 cases). Three distal duplications of $16 \mathrm{p} 11.2$ were found in ADHD, ASD, and SCZ cases. We found 15q11-q13 duplications in six cases diagnosed with ADHD, ASD, OCD, or SCZ. The high prevalence of $15 q 11.2$ duplications (BP1-BP2) and 16p13.11 duplications likely reflects the relatively mild expression and reduced penetrance of these genotypes.

Fourth, we identified de novo CNVs impacting genes in 35 cases: $31 / 448$ (6.9\%) for ASD and 3/167 (1.8\%) for OCD (category D in Tables 2, 3). Of $13 \mathrm{SCZ}$ trios with data, we identified one de novo genic CNV. Parents of ADHD cases were not analyzed, thus no data on de novo CNVs were available for this disorder.

The last class of variants included inherited clinically relevant CNVs that did not belong to any of the first three categories. These included CNVs impacting genes previously implicated for NDDs but either inherited or with unknown inheritance. We found these variants in $117 / 2,691$ cases (4.4\%) (category $E$ in Tables 2, 3).

Cases with more than one clinically relevant CNV

We found more than one clinically relevant CNV in $20 / 2,691$ $(0.74 \%)$ of NDD cases: $12 / 1,838(0.65 \%)$ of ASD cases, $4 / 427$ $(0.95 \%)$ of ADHD cases; $3 / 222$ (1.35\%) of those with OCD, and $1 /$ $204(0.5 \%)$ of individuals with SCZ.

In ASD, there were eleven cases with two CNVs and one case with three relevant variants. The latter, case 4-0040-003, had a maternally inherited $53 \mathrm{~kb}$ deletion impacting CTNNA3, a maternally inherited $54 \mathrm{~kb}$ duplication impacting CNTN4, and a de novo $547 \mathrm{~kb}$ duplication impacting YES1, ADCYAP1, and six other genes. Examples with two clinically relevant CNVs each included: (i) a male (7-0293-003) with a $611 \mathrm{~kb}$ deletion of $16 \mathrm{p} 11.2$ and a $513 \mathrm{~kb}$ deletion of $15 q 11.2$, of unknown inheritance; (ii) a female (2-1525003) with a maternally inherited $1.5 \mathrm{Mb}$ deletion consistent with the 16p13.11 recurrent microdeletion (neurocognitive disorder susceptibility locus), and a de novo $326 \mathrm{~kb}$ duplication impacting USP7, PMM2, C16orf72, and CARHSP1; and (iii) a male (2-0305-004) with a maternally inherited $432 \mathrm{~kb}$ deletion in the 1q21.1 locus associated with thrombocytopenia-absent radius syndrome and a de novo $1.09 \mathrm{Mb}$ duplication impacting GPD2 and NR4A2.
The four cases with ADHD with more than one CNV were all male; each had two clinically relevant CNVs, all of unknown inheritance. Examples included: (i) 213050 with Klinefelter syndrome $(47, \mathrm{XXY})$ and an autosomal $181 \mathrm{~kb}$ duplication affecting MCPH1; (ii) 206760 with a $191 \mathrm{~kb}$ deletion impacting DLGAP2 and a $154 \mathrm{~kb}$ duplication involving DRD4 and eight more genes; and (iii) $235983 \mathrm{~S}$ with Klinefelter syndrome $(47, \mathrm{XXY})$ and an autosomal $123 \mathrm{~kb}$ duplication impacting DPP6.

Of the three OCD cases carrying more than one CNV, there was one male (OCD146-JS-1254_188613) with three clinically significant CNVs: a maternally inherited $143 \mathrm{~kb}$ deletion impacting NLGN1, a second maternally inherited $115 \mathrm{~kb}$ deletion impacting $D P P 6$, and a paternally inherited $67 \mathrm{~kb}$ duplication impacting PTPRN2. Another male (OCD125-896993) had a de novo $165 \mathrm{~kb}$ deletion impacting $A D R A 2 \mathrm{C}$ and a paternally inherited $1.7 \mathrm{Mb}$ duplication impacting CNTN4 and CNTN6. A female (OCD109-1648) had a maternally inherited $1.4 \mathrm{Mb}$ deletion of $17 \mathrm{p} 12$ and a $190 \mathrm{~kb}$ deletion impacting PTPRT. This case also had a de novo frameshift deletion in LRCH2 (c.2190_2193del:p.C730fs; Supplementary Table $1 C$ ) identified using WES.

The only individual with SCZ having two clinically relevant CNVs (Supplementary Table 1C) was a female (222720) with a $10.2 \mathrm{Mb}$ deletion of chromosome $5 p$ congruent with the cri-du-chat syndrome region, and a $7.3 \mathrm{Mb}$ duplication of 6q26-27. Karyotyping confirmed this to be the result of an unbalanced translocation. ${ }^{11}$

Cases with clinically relevant CNVs identified by microarray also had SNVs or CNVs detected by WGS or WES

No WGS data were available for the ADHD and SCZ samples. However, we have previously published WGS data for 106/209 (50.7\%) ASD cases with clinically relevant CNVs. ${ }^{8,27,28}$ Of these, 15 had a clinically relevant LoF mutation and one had a $4.5 \mathrm{~kb}$ deletion (Supplementary Table 1C). The latter case was a male (21086-004) with a maternally inherited $80.1 \mathrm{~kb}$ duplication impacting CTNND2, identified using array data. He also had paternally inherited $4.5 \mathrm{~kb}$ deletion in $A N O 3$, known to be associated with autosomal dominant dystonia (OMIM: 610110). This deletion was missed by microarray for lack of probes in this region, due to its size (Supplementary Table 1C). Examples of LoF SNVs were: (i) a female autism case (7-0133-003) with a $2.5 \mathrm{Mb}$ de novo duplication in 10q11.22-11.23 and a de novo nonsense mutation in SOX5 (c.C313T:p.R105X). Mutations of SOX5 cause autosomal dominant Lamb-Shaffer syndrome, characterized by global developmental delay and intellectual disability (OMIM:604975), and (ii) a male autism case (7-0123-003) with a $2.9 \mathrm{Mb}$ duplication involving 
Table 3. Copy number variations of clinical significance among four neurodevelopmental disorders

\begin{tabular}{|c|c|c|c|c|c|c|c|c|}
\hline Category & $\#^{1}$ & Cytoband & Type & Size (kb) & Sex & Disorder & Class $^{2}$ & Origin \\
\hline \multicolumn{9}{|l|}{ A: aneuploidies $(n=17)$} \\
\hline $45, x$ & 2 & Xp22.33-q28 & DEL & 155,271 & $\mathrm{~F}$ & ASD, ADHD, SCZ ${ }^{\mathrm{A}}$ & CS & $D, U$ \\
\hline $47, X X,+21 / 47, X Y,+21$ & 6 & $21 p 13-q 22.3$ & DUP & 48,130 & $F(2), M(4)$ & ASD & CS & $\mathrm{D}, \mathrm{U}(5)$ \\
\hline $47, X Y Y$ & 4 & Yp11.32-q12 & DUP & 59,374 & M & $\operatorname{ASD}(2), A D H D(2), S C Z^{A}$ & CS & $\mathrm{D}(2), \mathrm{U}(2)$ \\
\hline \multicolumn{9}{|c|}{ B: large CNVs ( $>3 \mathrm{Mb} ; n=23$ ) excluding aneuploidies (category A) and CNVs associated with known recurrent genomic disorders (category C) } \\
\hline $46, X Y, \operatorname{dup}(4)(q 22.1 q 22.2)$ & 1 & $4 q 22.1-q 22.2$ & DUP & 3,060 & M & ASD & VUS & u \\
\hline $46, \mathrm{XY}, \mathrm{del}(4)(\mathrm{q} 32.3)$ & $2^{*}$ & $4 q 32.3$ & DEL & 3,209 & M & ASD & VUS & $u$ \\
\hline $46, X X$,dup(4)(q34.3q35.1) & 1 & $4 q 34.3-q 35.1$ & DUP & 3,886 & $\mathrm{~F}$ & $\mathrm{SCZ}, \mathrm{SCZ}{ }^{\mathrm{A}}$ & VUS & $u$ \\
\hline 46,XY,dup(6)(q15q16.1) & 1 & $6 q 15-q 16.1$ & DUP & 4,739 & M & ASD & LCS & M \\
\hline $46, X X$,dup,(6)(p26q27) & 1 & $6 p 26-q 27$ & DUP & 7,302 & $\mathrm{~F}$ & $\mathrm{SCZ}, \mathrm{SCZ} \mathrm{A}^{\mathrm{A}}$ & LCS & $u$ \\
\hline $46, X Y, \operatorname{del}(7)(q 31.1 q 31.31)$ & 1 & $7 q 31.1-q 31.31$ & DEL & 11,051 & M & ASD, SCZ ${ }^{A}$ & CS & $\mathrm{D}$ \\
\hline 46,XY,del(9)(p22.1p21.2) & 1 & $9 p 22.1-p 21.2$ & DEL & 8,005 & M & ASD & CS & $u$ \\
\hline $46, X Y$, del $(13)(q 14.13 q 14.3)$ & 1 & $13 q 14.13-q 14.3$ & DEL & 5,350 & M & SCZ & LCS & $u$ \\
\hline 46,XY,dup(15)(q25.3q26.1) & 1 & $15 q 25.3-q 26.1$ & DUP & 6,519 & M & ASD & LCS & $u$ \\
\hline $46, \mathrm{XY}, \mathrm{dup}(16)(q 11.2 q 21)$ & 1 & $16 q 11.2-q 21$ & DUP & 15,626 & M & ASD & $\mathrm{CS}$ & $u$ \\
\hline $46, X Y, \operatorname{dup}(16)(q 11.2 q 13)$ & 1 & $16 q 11.2-q 13$ & DUP & 10,873 & M & ASD & CS & D \\
\hline $46, X X$,dup(17)(q11.1q12) & 1 & $17 q 11.1-q 12$ & DUP & 6,145 & $\mathrm{~F}$ & ASD & CS & u \\
\hline 46,XY,del(18)(p11.32p11.21) & 1 & $18 p 11.32-p 11.21$ & DEL & 15,264 & M & ASD & CS & $u$ \\
\hline $46, X Y, \operatorname{del}(18)(q 22.1 q 23)$ & 1 & $18 q 22.1-q 23$ & DEL & 13,469 & M & ASD & CS & $u$ \\
\hline $46, X X, \operatorname{dup}(22)(q 11.21 q 12.3)$ & 1 & $22 q 11.21-q 12.3$ & DUP & 12,518 & $\mathrm{~F}$ & ADHD & CS & $u$ \\
\hline $46, \mathrm{XY}, \operatorname{del}(\mathrm{Y})(\mathrm{p} 11.2)$ & 1 & Yp11.2 & DEL & 3,140 & M & ADHD & VUS & $\mathrm{U}$ \\
\hline \multicolumn{9}{|c|}{ C: CNVs associated with known recurrent genomic disorders $(n=115)$} \\
\hline 1q21.1 Proximal Deletion (TAR Syndrome) & 3 & $1 \mathrm{q} 21.1$ & DEL & $410-513$ & M & ASD & VUS & $M(2), U$ \\
\hline 9q34.3 Duplication & 1 & $9 q 34.3$ & DUP & 2,064 & M & ASD & LCS & u \\
\hline 10q11.22-q11.23 Duplication & $2^{*}$ & $10 q 11.22-q 11.23$ & DUP & $2,650-2,661$ & $\mathrm{~F}$ & ASD, SCZ ${ }^{\mathrm{A}}$ & vUs & $\mathrm{D}$ \\
\hline 15q11-q13 Duplication & 6 & $15 q 11.2-q 13.1$ & DUP & $4,918-6,158$ & $F(2), M(4)$ & $\begin{array}{l}\mathrm{ADHD}, \mathrm{ASD}(3), \mathrm{OCD}, \mathrm{SCZ}, \\
\mathrm{SCZ}^{\mathrm{A}}(6)\end{array}$ & CS & $u$ \\
\hline 15q11.2 Deletion (BP1-BP2) & 16 & $15 q 11.2$ & DEL & $312-521$ & $F, M(15)$ & $\operatorname{ADHD}(3), \operatorname{ASD}(12), \mathrm{OCD}$ & VUS & $M(4), U(12)$ \\
\hline 15q11.2 Duplication (BP1-BP2) & 15 & $15 q 11.2$ & DUP & $311-850$ & $F(7), M(8)$ & $\operatorname{ADHD}(3), \operatorname{ASD}(12)$ & LB & $\begin{array}{l}M(2), P(2), U \\
(11)\end{array}$ \\
\hline 15q13.3 Deletion & 4 & $15 q 13.2-q 13.3$ & DEL & $1,533-2,072$ & $F(2), M(2)$ & ASD, SCZ ${ }^{A}$ & CS & $\mathrm{P}, \mathrm{U}(3)$ \\
\hline 15q25 Distal Deletion & 1 & $15 q 25.2-q 25.3$ & DEL & 959 & $\mathrm{~F}$ & ASD & VUS & U \\
\hline Rubinstein-Taybi Syndrome & 1 & $16 p 13.3$ & DUP & 204 & M & ASD & vUS & $u$ \\
\hline 16p13.11 Deletion & 3 & 16p13.11-p12.3 & DEL & $1,496-3,352$ & $\mathrm{~F}, \mathrm{M}(2)$ & $\mathrm{ADHD}, \mathrm{ASD}(2)$ & CS, VUS(2) & $\mathrm{D}, \mathrm{M}, \mathrm{U}$ \\
\hline 16p13.11 Duplication & 17 & 16p13.11-p12.3 & DUP & $783-2,950$ & $F(3), M(14)$ & $\begin{array}{l}\mathrm{ADHD}, \mathrm{ASD}(12), \mathrm{OCD}, \mathrm{SCZ}(3), \\
\mathrm{SCZ}^{\mathrm{A}}\end{array}$ & vUS & $\begin{array}{l}M(3), P(3), U \\
(11)\end{array}$ \\
\hline 16p12.1 Duplication & 3 & $16 \mathrm{p} 12.2$ & DUP & $670-680$ & $F, M(2)$ & ASD & Vus & $M(2), U$ \\
\hline 16p12.1 Deletion & 3 & $16 \mathrm{p} 12.2$ & DEL & $613-655$ & M & $\operatorname{ADHD}(2), \mathrm{ASD}$ & vUs & $u$ \\
\hline 16p11.2 Distal Duplication & 3 & $16 \mathrm{p} 11.2$ & DUP & $273-362$ & $F, M(2)$ & ADHD, ASD, SCZ & CS & $U$ \\
\hline 16p11.2 Distal Deletion & 2 & $16 p 11.2$ & DEL & $227-243$ & $\mathrm{~F}, \mathrm{M}$ & ASD & CS & $\mathrm{D}, \mathrm{U}$ \\
\hline 16p11.2 Proximal Duplication & 1 & $16 \mathrm{p} 11.2$ & DUP & 625 & M & $\mathrm{ADHD}, \mathrm{ADHD}^{\mathrm{A}}, \mathrm{SCZ}^{\mathrm{A}}(4)$ & CS & $\mathrm{u}$ \\
\hline 16p11.2 Proximal Deletion & 4 & $16 \mathrm{p} 11.2$ & DEL & $598-746$ & $F, M(3)$ & $\mathrm{ADHD}, \mathrm{ASD}(2), \mathrm{SCZ}, \mathrm{SCZ} \mathrm{Z}^{\mathrm{A}}$ & CS & $u$ \\
\hline 17p12 Deletion & 1 & $17 p 12$ & DEL & 1,404 & $\mathrm{~F}$ & OCD & CS & M \\
\hline 17p12 Duplication & 2 & $17 q 12$ & DUP & $1,858-1,970$ & M & ADHD, ASD & CS & $u$ \\
\hline 22q11.21 Deletion & 7 & $22 q 11.21$ & DEL & $1,396-3,154$ & $F(3), M(4)$ & $\mathrm{ASD}, \mathrm{SCZ}(6), \mathrm{SCZ}^{\mathrm{A}}(6)$ & CS & $u$ \\
\hline 22q11.21 Duplication & 7 & $22 q 11.21$ & DUP & $2,546-3,271$ & M & ASD & CS & $D, P, U(5)$ \\
\hline
\end{tabular}




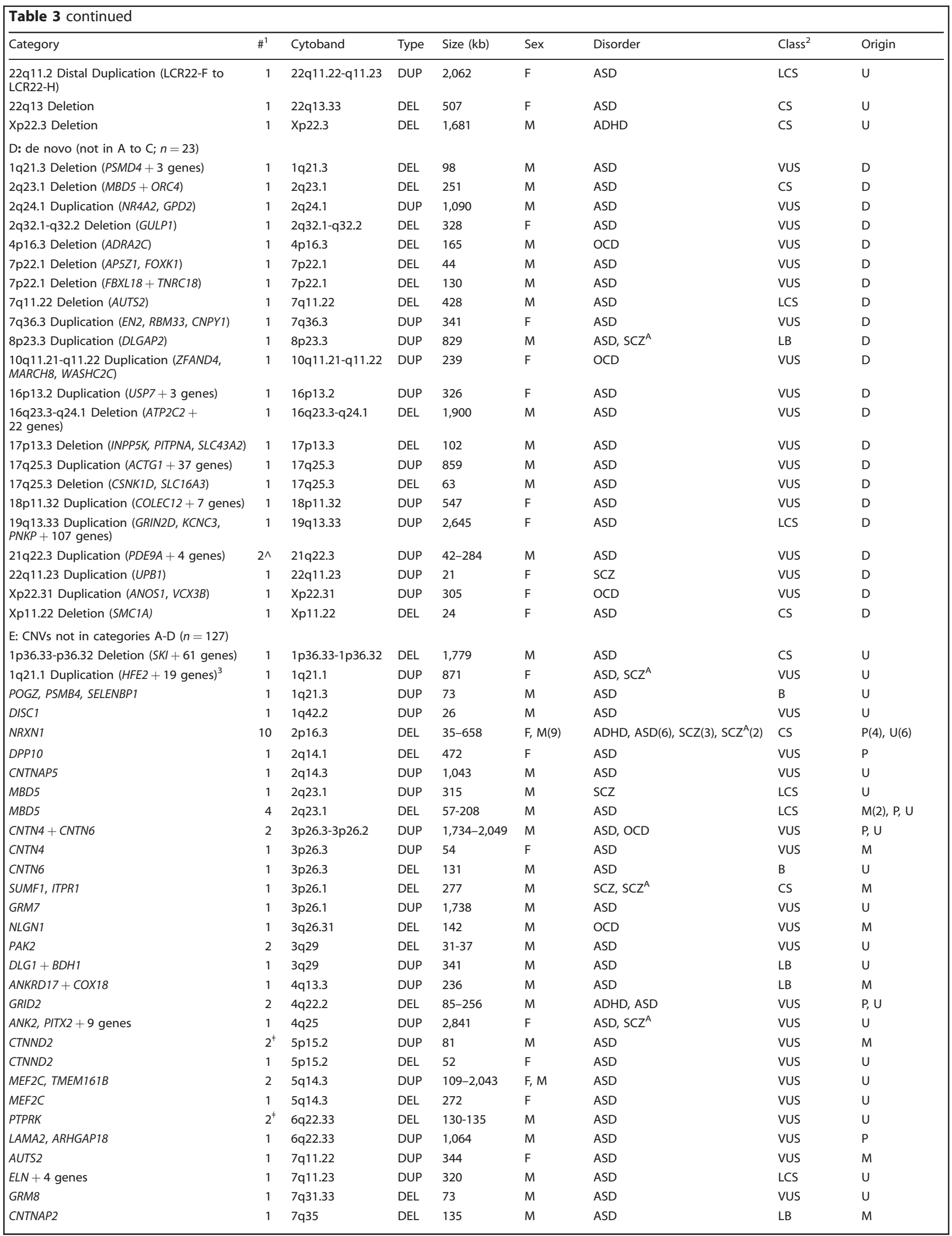


Table 3 continued

\begin{tabular}{|c|c|c|c|c|c|c|c|c|}
\hline Category & $\#^{1}$ & Cytoband & Type & Size $(k b)$ & Sex & Disorder & Class $^{2}$ & Origin \\
\hline KMT2C & 1 & $7 q 36.1$ & DUP & 440 & $\mathrm{~F}$ & ASD & LB & $U$ \\
\hline DPP6, PAXIP1, HTR5A & 3 & $7 q 36.2$ & DUP & $123-1,800$ & $M$ & $\mathrm{ADHD}, \mathrm{ASD}(2)$ & VUS & $M, U(2)$ \\
\hline DPP6 & 3 & $7 q 36.2$ & DEL & $52-396$ & M & $\mathrm{ADHD}, \mathrm{ASD}, \mathrm{OCD}, \mathrm{SCZ} \mathrm{Z}^{\mathrm{A}}$ & Vus & $M, P, U$ \\
\hline PTPRN2, ESYT2, NCAPG2 & 3 & $7 q 36.3$ & DUP & $50-430$ & M & $A D H D, A D H D^{A}, A S D, O C D$ & LB & $M, P, U$ \\
\hline DLGAP2, CLN8, ARHGEF10 & 3 & $8 p 23.3$ & DUP & $317-358$ & M & ASD & VUS & $\mathrm{P}$ \\
\hline DLGAP2 & 2 & $8 p 23.3$ & DEL & $58-191$ & M & ADHD, ASD & VUS & $\mathrm{U}$ \\
\hline $\mathrm{MCPH} 1$ & 2 & $8 p 23.2-8 p 23.1$ & DUP & $181-272$ & M & ADHD, OCD & LB & $M, U$ \\
\hline PTPRD & 1 & $9 p 24.1$ & DEL & 81 & M & ASD & VUS & $U$ \\
\hline ASTN2, TRIM32, PAPPA & 3 & $9 q 33.1$ & DEL & $25-523$ & $F, M(2)$ & $\operatorname{ADHD}^{A}(2), A S D$ & VUS & $U$ \\
\hline PCDH15 & 2 & $10 q 21.1$ & DEL & $58-291$ & $\mathrm{~F}, \mathrm{M}$ & ASD & VUS & $\mathrm{U}$ \\
\hline CTNNA3 & 7 & $10 q 21.3$ & DEL & $53-306$ & $F(2), M(5)$ & ASD & B & $M(3), P, U(3)$ \\
\hline 10q24.32 Duplication (POLL, BTRC, DPCD) & 1 & $10 q 24.32$ & DUP & 226 & M & ASD & LCS & $\mathrm{P}$ \\
\hline$D R D 4+8$ genes & 1 & $11 \mathrm{p} 15.5$ & DUP & 154 & M & ADHD & VUS & $U$ \\
\hline PAX6, ELP4 & 1 & $11 \mathrm{p} 13$ & DUP & 393 & M & ADHD & VUS & $U$ \\
\hline SHANK2 & 1 & $11 q 13.4$ & DEL & 132 & $M$ & ASD & LCS & $M$ \\
\hline$D L G 2$ & 1 & $11 q 14.1$ & DEL & 210 & $\mathrm{~F}$ & ASD & VUS & $U$ \\
\hline CNTN5 & 1 & $11 q 22.1$ & DUP & 25 & $M$ & ASD & VUS & $U$ \\
\hline$K M T 2 A+6$ genes & 1 & $11 q 23.3$ & DUP & 265 & $\mathrm{~F}$ & ASD & VUS & M \\
\hline CACNA1C & 1 & $12 p 13.33$ & DUP & 56 & $M$ & ASD & VUS & $P$ \\
\hline PCDH9 & 1 & $13 q 21.32$ & DUP & 1,006 & M & ASD & VUS & M \\
\hline CHD8 & 1 & $14 q 11.2$ & DUP & 23 & M & ASD & VUS & $P$ \\
\hline NRXN3 & $3^{ \pm}$ & $14 q 31.1$ & DEL & $224-254$ & $\mathrm{~F}, \mathrm{M}(2)$ & ASD & LCS & $P(2), U$ \\
\hline $\begin{array}{l}\text { 15q13.1-q13.2 Duplication (APBA2 + } \\
4 \text { genes) }\end{array}$ & 1 & $15 q 13.1-q 13.2$ & DUP & 1,376 & $\mathrm{M}$ & ASD & VUS & M \\
\hline CHRNA7 & 1 & $15 q 13.3$ & DEL & 441 & $\mathrm{~F}$ & ASD & LCS & $\mathrm{P}$ \\
\hline RBFOX1 & 1 & $16 p 13.3$ & DEL & 103 & $\mathrm{~F}$ & $\mathrm{ASD}, \mathrm{SCZ}^{\mathrm{A}}(2)$ & VUS & U \\
\hline RBFOX1 & 2 & $16 p 13.3$ & DUP & $42-373$ & M & ASD & VUS & $U$ \\
\hline 16p11.2 Duplication (ATP2A1 + 20 genes) & 1 & $16 \mathrm{p} 11.2$ & DUP & 962 & $\mathrm{~F}$ & ASD & VUS & $\mathrm{U}$ \\
\hline ATP2C2 +11 genes & 1 & $16 q 24.1$ & DUP & 1,061 & $\mathrm{~F}$ & ASD & VUS & $U$ \\
\hline$A T P 2 C 2, T L D C 1$ & 3 & $16 q 24.1$ & DEL & $23-126$ & M & ADHD & LB & $U$ \\
\hline ANKRD11 + 5 genes & 1 & $16 q 24.3$ & DUP & 250 & M & ASD & VUS & $\mathrm{U}$ \\
\hline$N F 1+14$ genes & 3 & $17 q 11.2$ & DUP & $83-1,394$ & $\mathrm{~F}, \mathrm{M}(2)$ & ADHD, ASD, SCZ & LCS, VUS(2) & $P, U(2)$ \\
\hline DLGAP1 & 1 & $18 p 11.31$ & DUP & 62 & M & OCD & VUS & $\mathrm{P}$ \\
\hline $\mathrm{CDH7}, \mathrm{CDH} 19$ & 1 & $18 p 22.1$ & DEL & 2,126 & $\mathrm{~F}$ & OCD & VUS & M \\
\hline MACROD2 & 1 & $20 p 12.1$ & DUP & 22 & $M$ & ASD & VUS & $\mathrm{U}$ \\
\hline MACROD2 & 2 & $20 \mathrm{p} 12.1$ & DEL & $72-237$ & M & $\operatorname{ADHD}^{A}(2), A S D, O C D, S C Z^{A}$ & VUS & U \\
\hline PTPRT +6 genes & 1 & $20 q 12-q 13.12$ & DUP & 836 & M & $A S D, S C Z^{A}$ & VUS & $\mathrm{U}$ \\
\hline PTPRT & 1 & $20 q 12$ & DEL & 190 & $\mathrm{~F}$ & OCD & VUS & $U$ \\
\hline $\begin{array}{l}22 q 11.21 \text { Deletion (SNAP29, LZTR1 + } \\
12 \text { genes) }\end{array}$ & 1 & $22 q 11.21$ & DEL & 749 & $M$ & ADHD & LCS & $U$ \\
\hline CACNA11 & 1 & $22 q 13.1$ & DUP & 116 & $M$ & ASD & VUS & $U$ \\
\hline$A R S E+7$ genes & 2 & Xp22.33-Xp22.32 & DUP $^{4}$ & 2,262 & M & ASD & VUS & $P$ \\
\hline ANOS1 +4 genes & 1 & Xp22.31 & DUP & 1,247 & $\mathrm{~F}$ & OCD & VUS & U \\
\hline PTCHD1-AS + 5 genes & 5 & Xp22.11 & DEL & $81-1,169$ & $M$ & $\mathrm{ADHD}^{\mathrm{A}}, \mathrm{ASD}$ & VUS & $M(4), U$ \\
\hline ILIRAPL1 & 1 & $\mathrm{Xp} 21.3-\mathrm{Xp} 21.2$ & DUP & 546 & $M$ & ASD & LCS & M \\
\hline DMD, TAB3, FTHL17 & 2 & Xp21.2-Xp21.1 & DUP & $252-792$ & M & ASD & LCS,VUS & M \\
\hline$R A B 38 B+8$ genes & 1 & $\mathrm{Xq} 28$ & DUP & 286 & $\mathrm{~F}$ & ADHD & VUS & $\mathrm{U}$ \\
\hline
\end{tabular}

WBS Williams-Beuren Syndrome, $F$ female, $M$ male, DEL deletion, DUP duplication, $A S D$ autism spectrum disorder, $A D H D$ attention deficit hyperactivity disorder, $S C Z$ schizophrenia, OCD obsessive compulsive disorder, VUS variants of unknown significance, $B$ benign, $L B$ likely benign, CS clinically significant, $L C S$ likely clinically significant, $M$ maternally inherited, $P$ paternally inherited, $U$ unknown inheritance, $D$ de novo. ${ }^{1}$ Number of subjects carrying CNVs in the indicated cytoband. ${ }^{2}$ Clinical impact of variants was evaluated according to accepted clinical guidelines. ${ }^{3}$ Mosaic CNV. ${ }^{4}$ These two duplications are in two brothers. qPCR showed that the father was also carrying these duplications on chromosome $X$, which implies that there might be translocation between $X$ and an unknown autosome, which then was transmitted to these two boys. We did not attempt identify the location of this duplication on autosomes. ${ }^{\mathrm{A}}$ Indicates the presence of a similar CNV detected in a subject published previously as part of two large-scale schizophrenia CNV studies (Costain et al. ${ }^{24}$ and Lowther et al. ${ }^{11}$ ) and one ADHD study (Lionel et al. ${ }^{10}$ ). Sex, size of CNVs, inheritance, and the clinical classifications of these CNVs were not provided

Only genes whose coding sequences are impacted by CNVs are shown. However, CNVs impacting exons (UTRs) in MBD5 and PTCHD-AS were indicated ${ }^{*}$ Monozygotic pair of twins from two different families; ${ }^{*}$ Siblings; $\wedge$ two duplications from one case. ${ }^{ \pm}$two of these deletions are paternally inherited in siblings diagnosed with ASD (one male, one female)

The coordinates for clinically relevant CNVs are in Supplementary Table $1 \mathrm{C}$ 
a) aneuploidies

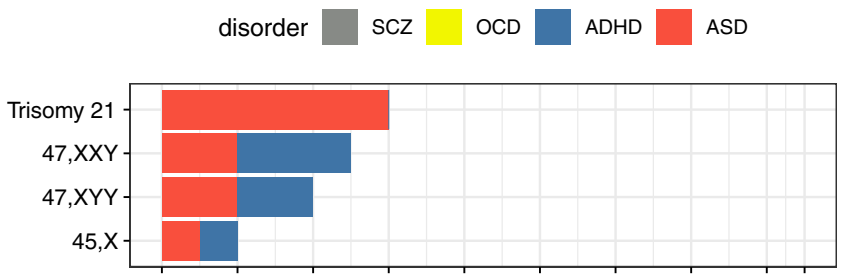

b) recurrent loci

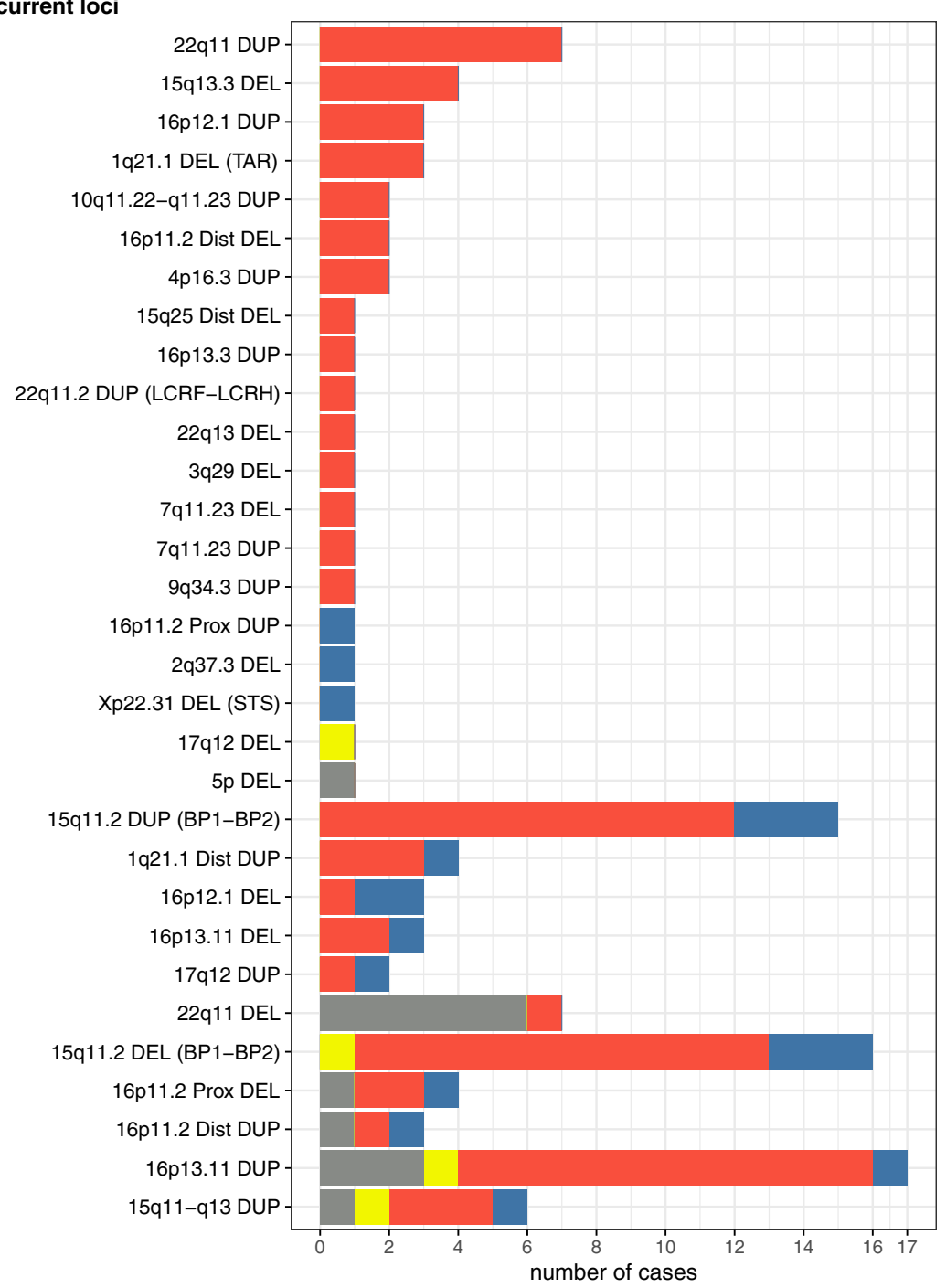

Fig. 1 Distribution of a) aneuploidies and b) known recurrent genomic disorder CNVs found in cases diagnosed with autism spectrum disorder (ASD), attention deficit hyperactivity disorder (ADHD), schizophrenia (SCZ), or obsessive compulsive disorder (OCD). Details of the copy number variants, sex, and variant sizes are in Table 1, Supplementary Table 1A. DEL deletion, DUP duplication, TAR ThrombocytopeniaAbsent Radius syndrome locus, STS includes STS, BP breakpoint, LCR low-copy repeat, Prox proximal, Dist distal

the $16 \mathrm{p} 13.11$ recurrent microduplication who also had a de novo splice-site variant impacting SHANK3 (c.2223 $+1 \mathrm{G}>\mathrm{A})$, which is a gene strongly associated with NDDs. ${ }^{8}$ Of 13 OCD cases with clinically relevant CNVs, three also had clinically relevant LoF mutations previously identified using WES (Supplementary Table 1C). ${ }^{13}$ One example is a male OCD case (OCD146-JS-1254_188613) with three clinically relevant CNVs: a maternally inherited $142 \mathrm{~kb}$ deletion in $3 q 26.31$, a maternally inherited $115 \mathrm{~kb}$ deletion in $7 q 36.2$, and a paternally inherited $67 \mathrm{~kb}$ duplication in 7q36.3. He also had three clinically relevant SNVs found by WES: (i) a maternally inherited frameshift deletion in AFF2 (c.2976_2988del: p.992_996del), which is an X-linked recessive variant associated with mental retardation (OMIM:300806), (ii) a maternally inherited frameshift deletion in DRD4 (c.233_245del:p.A78fs), an autosomal dominant variant associated with autonomic nervous system dysfunction and ADHD (OMIM:126452), and (iii) a maternally inherited frameshift in MBD4 (c.939_940ins:p.E314fs), a gene involved in DNA methylation (OMIM:603574).

\section{Complex phenotypes}

Because we had clinical information on NDD phenotypes beyond the primary diagnoses for some cases, we investigated the pleiotropy of CNVs shared among different NDDs (Supplementary 


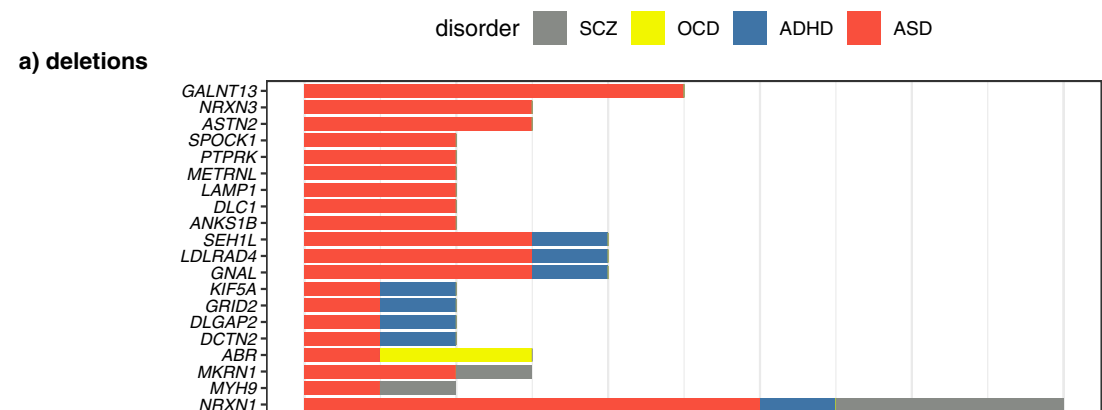

b) duplications

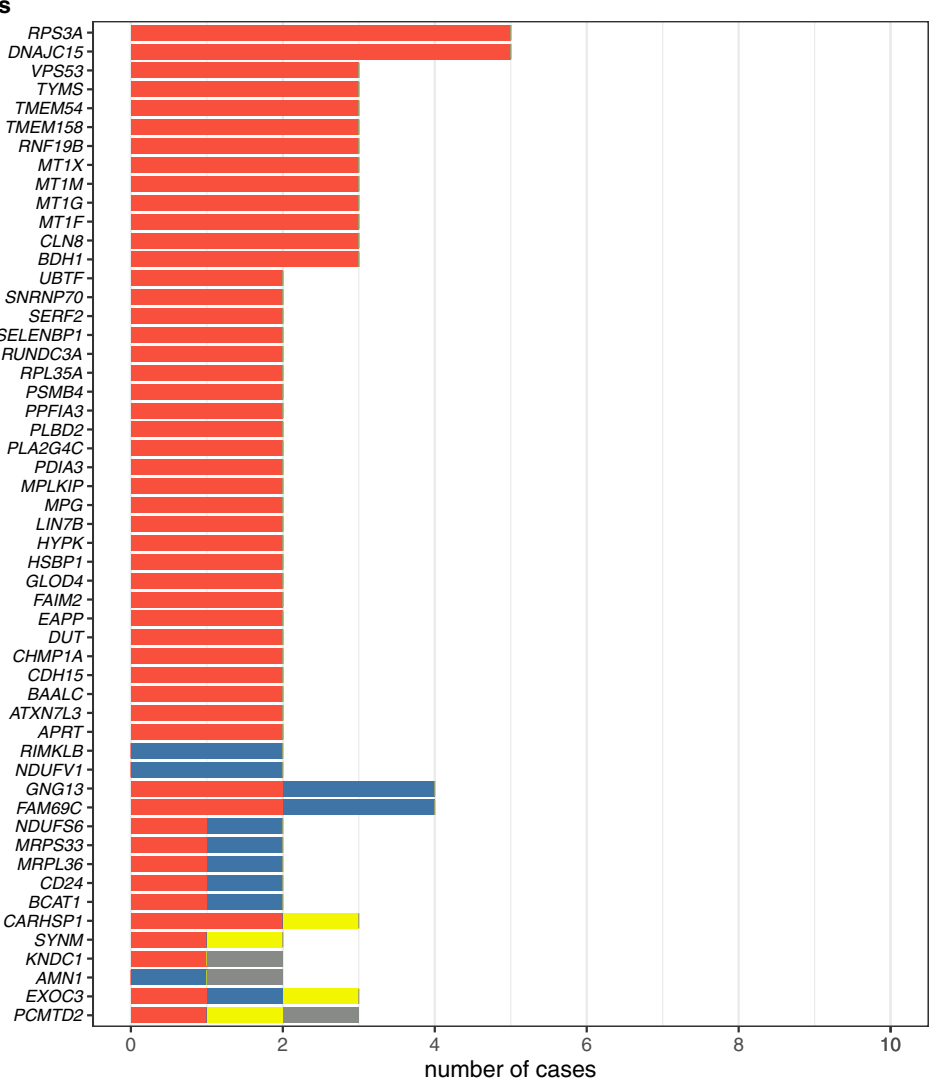

Fig. 2 Genes impacted by rare CNVs in more than one case. a) brain-expressed and moderately constrained genes (pLI $>0.45$ ) impacted by deletions in multiple cases, b) brain-expressed genes with duplication of their full-length transcript in more than one case

Table 1C). We defined complex as having multiple different NDDs. Of ADHD cases with an NDD relevant CNV, a few were noteworthy and highlight the clinical pleiotropy associated with many of these variants. A male ascertained for ADHD (176004), who also had a learning disability but no ASD, carried a duplication of 16p11.2, which is known to be associated with ASD. ${ }^{8}$ We found deletions at this locus in a female with SCZ, a male with ASD, and another male with $A D H D$, but none in our OCD cases. A male diagnosed with ADHD (206773), who carried a duplication of chromosome $X$ (Klinefelter syndrome), also had ASD, learning disability, language delay, general anxiety disorder, and enuresis, all known feature of Klinefelter syndrome. ${ }^{29}$ Male case 181220 with ADHD, with 15q11.2 duplication (BP1-BP2), also had ASD. Of OCD cases, a male (OCD75-SB-1213) with a paternally inherited $62 \mathrm{~kb}$ duplication of DLGAP2 also had separation anxiety disorder, a Tourette disorder with tic, oppositional defiant disorder, and panic disorders and agoraphobia. Another male with OCD (OCD146-JS1254_188613), ADHD (inattentive subtype), and a Tourette disorder with tic, had three different CNVs, impacting NLGN1,
DPP6, and PTPRN2. Of SCZ cases, a male (153030) with a $1.6 \mathrm{Mb}$ duplication of $16 \mathrm{p} 13.11$ also had a learning disorder but no ID (details in Supplementary Table 1C). A female (213684) with SCZ and a $549 \mathrm{~kb}$ deletion of NRXN1 also had moderate intellectual disability. A male with SCZ (166808) with a 15q11-q13 duplication had mild intellectual disability.

Cross-disorder gene discovery and genes in multiple cases in a single disorder

We searched for genes, excluding those from regions of known recurrent genomic disorders, that were affected in multiple cases by CNVs. We first restricted analysis to brain-expressed genes that are at least moderately constrained for LoF variants, ( $\mathrm{pLI}>0.45$; Fig. 2; Supplementary Table 1D). We searched these for genes impacted by CNVs in at least two cases each, and found 20 genes impacted by deletions. Notably, NRXN1 was impacted in 10 subjects (three SCZ, six ASD, and one ADHD); deletions of 18p11.21 impacting novel candidate genes (GNAL, LDLRAD4, and SEH1L) were in four cases (three ASD, one ADHD). Genes MKRN1 and 
MYH9 involved CNVs in ASD and SCZ cases. Eight genes - NRXN1, GNAL, LDLRAD4, SEH1L, DLGAP2, DCTN2, GRID2, and KIF5A involved CNVs in ASD and ADHD cases. Only CNVs containing $A B R$ were shared between $O C D$ and $A S D$, and no gene-containing variants were shared between $O C D$ and $A D H D$ or OCD and SCZ.

Genes impacted by deletions in multiple ASD cases (only) were: ASTN2, NRXN3, ANKS1B, GALNT13, DLC1, LAMP1, METRNL, PTPRK, and SPOCK1 (Fig. 2; Supplementary Table 1D). Although ASTN2 deletions were previously reported in $\mathrm{ADHD}^{30}$ in this study we made no such observations. We saw no genes impacted by deletions in multiple cases of ADHD, OCD, or SCZ, other than those in the known genomic syndromes (Fig. 2; Supplementary Table 1E).

We identified 53 brain-expressed genes impacted in multiple cases by duplications of the entire longest transcript (Fig. 2; Supplementary Table 1E). Examples included DNAJC15, GNG13, CARHSP1, PCMTD2, RPS3A, and TMEM158 (Supplementary Table $1 \mathrm{E})$. Most whole-gene duplications were from ASD cases, probably due to the latter's disproportionate representation. Examples of genes with such variants in multiple disorders were: PCMTD2 in ASD, OCD and SCZ; KNDC1 in ASD and SCZ; CARHSP1, PCMTD2, SYNM, and EXOC3 in both ASD and OCD; and GNG13, MRPS33, RPS3A, FAM69C, and CD24 in ASD and ADHD.

We found 38 genes duplicated in multiple ASD cases (only). Examples included CDH15, UBTF, DUT, HYPK, ATXN7L3, and GLOD4. Duplication of NDUFV1 and RIMKLB were each observed in two ADHD cases (Fig. 2; Supplementary Table 1E). We found no repeated full gene duplications in OCD or SCZ cases in this collection.

Increased burden of rare CNVs impacting brain-expressed protein coding genes and brain-expressed long non-coding RNA (IncRNA) We sought rare CNVs impacting exons of IncRNAs and found these in 1,130/2,691 cases (42\%). Restricting to brain-expressed IncRNAs, only $234 / 2,691$ cases $(8.7 \%)$ carried such rare CNVs. We tested for the extent to which the protein coding genes and IncRNAs were impacted by rare CNVs in cases compared with parents of cases. We found a nearly significant excess in cases over controls of deletions in protein-coding genes $(p=0.08$; false discovery rate $(F D R)=0.21)$, but not for IncRNAs $(p>0.1)$. We found no global excess burden of duplications for protein-coding genes and IncRNAs $(p>0.1)$. However, when focused on brain-expressed elements, we observed a modest increase of rare deletions impacting both protein-coding genes $(p=0.03$; FDR $=0.19)$ and IncRNAs ( $p=0.06$; FDR $=0.21$ ). We then performed a multivariate analysis to test whether the burden signal was from proteincoding, IncRNAs, or both. This analysis showed a statistically significant signal $(p=0.02)$ for deletions impacting protein-coding genes, suggesting an overlap between the protein-coding and IncRNA burden signal.

Given the increasing association of IncRNAs in disease, we highlight two example of such genes identified in multiple unrelated individuals. (i) AK127244: three subjects with ASD (10045-004, 7-0103-003, and 1-0629-003) harbored 2p16.3 deletions that directly disrupted the exonic sequence AK127244 (LOC730100). This is a $1.38 \mathrm{Mb}$ non-coding RNA of unknown function adjacent to NRXN1 and transcribed in the opposite direction. Rare, inherited deletions intragenic to AK127244 have been identified in five individuals with ASD. Such deletions have been proposed as candidate factors for a broad range of neuropsychiatric disorders including SCZ and affective disorder. ${ }^{16,31,32}$ We identified seven additional subjects here, five with ASD and two with SCZ, with coding deletions of NRXN1 that extend and disrupt the transcription start site and exonic sequence of AK127244. (ii) PTCHD1-AS: we found five males with ASD and deletions impacting exons of this gene (Table 3,
Supplementary Table 1C). Disruption of PTCHD1-AS has been linked to ASD. ${ }^{33,34}$

Increased burden of CNVs impacting NDD genes in cases carrying CNVs that impact genomic instability genes and fragile sites

We hypothesized that CNVs affecting genes involved in genome stability might lead to a higher incidence of additional variants. These subsequent variants could then add to the phenotypic complexity, by impacting genes involved in the development and functions of the nervous system. We therefore tested if individuals carrying a CNV that impacts "genomic instability genes" (GIG-CNV) have a higher burden of rare CNVs (measured as the number and the cumulative length of rare CNVs per individual) than do individuals not carrying such CNVs. ${ }^{35}$ We compiled a set of 958 protein coding "genomic instability genes" from the AmiGO database. ${ }^{36}$

The "genomic instability genes" were not disproportionately impacted by rare CNVs in cases compared with controls (parents or unaffected individuals for this analysis) $(p>0.1)$. In individuals who had a GIG-CNV, we found an increase in mean number of CNVs $\left(3.3\right.$ vs $\left.2.1 ; p=2.11 \times 10^{-8}\right)$ and cumulative length of rare CNVs (4.4 Mb vs $315 \mathrm{~kb} ; p=2.11 \times 10^{-8}$ ) compared to individuals without a GIG-CNV. We observed a similar trend excluding CNVs impacting the "genomic instability genes" from the burden analysis (mean number of rare CNVs: 2.8 vs $2.1 ; p=0.003$; cumulative length of rare CNVs: $564 \mathrm{~kb}$ vs $315 \mathrm{~kb} ; p=0.024)$. This difference was even higher when considering only cases (i.e. not controls)(mean number of rare CNVs: 2.9 vs 2.1; $p=0.013$; cumulative length of rare CNVs: $686 \mathrm{~kb}$ vs $358 \mathrm{~kb} ; p=0.051)$. We also found a 2.77-fold increase in the number of cases with rare CNVs impacting NDD-associated genes (NDD-CNV) $(n=1,160$; Supplementary Information) and a GIG-CNV (Fischer's exact test, $p=4.7 \times 10^{-05}$, odds ratio: $\left.2.77[\mathrm{Cl}: 1.66-4.54]\right)$, compared with cases with NDD-CNVs but without a GIG-CNV. We then excluded individuals with aneuploidy or a CNV impacting both "genomic instability genes" and NDD genes. We still observed the excess number of cases with NDD-CNVs and GIG-CNVs over those with NDD-CNVs only (odds ratio of 2.52[Cl:1.50-4.16] (Fisher's exact test, $\left.\left.p=2.7 \times 10^{-4}\right)\right)$

We then tested whether there was an increase CNV burden among cases whose parents had a GIG-CNV. Such CNVs could have been generated de novo anywhere previously in the pedigree and had not necessarily arisen de novo in the affected individual. We excluded the cases carrying a de novo GIG-CNV that was not found in the parents. We found a higher average number of rare CNVs in cases whose parents had GIG-CNVs compared to cases whose parents did not ( 2.66 vs $2.10 ; p=0.02$ ). We observed a similar trend for this global burden when excluding the GIG-CNVs (2.49 vs $2.10 ; p=0.08$ ). However, we did not observe an increased global burden in the cumulative length of rare CNVs.

We then further investigated cases with de novo CNVs for whom WGS data were available ( $n=16$, all from the ASD cohort). We found no over-representation $(p>0.1)$ of cases with de novo CNVs from families in which at least one parent carried a LoF variant impacting a "genomic instability gene" $(n=10)$ compared to other families $(n=6)$. Again, this was a small sample size. There were notable examples of cases with de novo CNVs whose parents had LoF variant(s) on "genomic instability genes". (i) Case 1-0627007 had a paternally inherited frameshift deletion impacting PALB2 (c.509_510del:p.R170fs). He also had a $1.9 \mathrm{Mb}$ de novo deletion in 16q23.3-q241 (Table 3, Supplementary Table 1C). (ii) Case 2-1525-003 had a stop-gain mutation on PALB2 (c.G2712A:p. W904X) and a $326 \mathrm{~kb}$ de novo duplication in 16p13.2. PALB2 plays a role in homologous recombination and checkpoint response. ${ }^{37}$ (iii) Case 1-0181-004 had a paternally inherited variant in EXO1 (c.G2482T:p.E828X) and a $5.3 \mathrm{Mb}$ de novo deletion in 3p14.1-p13. 
EXO1 functions in DNA replication, repair, and recombination (OMIM:606063). (iv) The father of ASD case 2-1693-003 had a RAD1 variant (c.168_172del:p.A56fs) - a gene required for DNA replication and repair (OMIM: 603153). She carried a de novo $24 \mathrm{~kb}$ deletion at $\mathrm{Xp11.22.}$

We also studied the extent of de novo CNVs overlapping genomic fragile sites. Of 33 de novo CNVs (excluding aneuploidies), 25 (75.6\%) overlapped fragile site regions (Supplementary Table 1F). In addition, eleven of the de novo CNVs overlapped long genes $(>300 \mathrm{~kb})$, a feature associated with fragile sites and neuronal genes. ${ }^{38}$ Notably, five of these genes - MBD5, FAM19A1, FOXP2, AUTS2, and DLGAP2 - are involved in neuron formation and differentiation.

\section{DISCUSSION}

We generated a bioresource to investigate the contribution of rare CNVs to the etiology of four NDDs - ASD, ADHD, OCD, and SCZ among 2,691 diagnosed cases. We found that $10.5 \%$ of these cases carried CNVs with potential clinical relevance to NDDs. Of all cases, $4.1 \%$ carried CNVs that were formally classified as clinically significant or likely clinically significant, when evaluated according to ACMG guidelines. ${ }^{26}$ We also found variant genes/regions that were shared across some or all of the NDDs. Evidence included recurrent or non-recurrent CNVs impacting the same genes in cases with different NDDs, and in patients diagnosed with multiple comorbid NDDs.

Of the four NDDs, ASD had the highest proportion of cases with a clinically relevant CNV $(11.4 \%)$. OCD cases had the lowest proportion with identified CNVs (5.6\%). Deletions of 22q11.21 were found in 6/204 SCZ cases (2.9\%) - three with mild intellectual disability - contributing to the relatively high proportion of SCZ cases deemed to have a clinically relevant CNV (10.8\%). 22q11.2 deletions are expected to be identified in about one in every 100-200 individuals with SCZ and about one in 10 with dual diagnosis of SCZ and ID. ${ }^{11}$ The enrichment of the SCZ cohort studied for ID likely contributed more to the prevalence observed of 22q11.2 deletions. Of ADHD cases, 9.4\% carried clinically relevant CNVs, which is slightly higher than $8.9 \%$ previously reported using a different microarray (Affymetrix SNP 6.0). ${ }^{10}$ We also found multiple clinically relevant CNVs in 20/2,691 (0.74\%) of NDD cases.

We found 17 aneuploidies $(45, \mathrm{X} ; 47, \mathrm{XXY}$ and $47, \mathrm{XYY})$ in cases diagnosed with either ASD or ADHD (Table 2). The prevalence of Turner syndrome $(45, X)(n=2)$ was $1 / 1,300$ among our cases, which is similar to previous reports. ${ }^{39}$ One had ADHD and the other ASD, similar to other reports. ${ }^{39}$ Cases with $47, \mathrm{XXY}(n=5)$ or $47, \mathrm{XYY}(n=4)$ had diagnoses of either ASD or ADHD, similar to previous reports. ${ }^{29}$ We found trisomy $21(n=6)$ only among ASD patients. ${ }^{40}$ Large CNVs other than aneuploidies were found in 23/ $2,691(0.85 \%)$ cases, mainly in gene-rich regions of the genome. Although we did not find aneuploidies in SCZ cases, they have been reported in association with this phenotype previously. ${ }^{11,24}$

We found CNVs associated with known recurrent genomic disorders in $4.3 \%$ of cases (Table 2). This signified an increase of this type of CNVs among NDDs compared with that from a community population (1.1\% (52/4,817); unpublished data), but similar to that of subjects with neurocognitive deficits in the UK Biobank (3.8\%). ${ }^{41}$

Known recurrent genomic disorders were distributed differently among the four NDDs (Table 2; Fig. 1). We observed ASD in 80/115 $(70 \%)$ of subjects with known recurrent genomic disorders, e.g., $7 q 11.23$ deletion, 16p11.2 distal deletion, and 22q11.21 duplications. The $5 p$ deletion was unique to SCZ. Deletions of $2 q 37.3$, $16 p 11.2$ proximal duplications, and $\mathrm{Xp} 22.3$ deletions were found only in ADHD, whereas $17 q 12$ deletion was only found in OCD. Duplications of 15q11-q13, deletions of 15q11.2 (BP1-BP2), and 16 p13.11 duplications were observed among cases of all four disorders (Fig. 1). Proximal duplications of 16p11.2 are found in up to $1 \%$ of individuals with SCZ. ${ }^{11,24,42}$

When parents were available to determine origin, we found $5.6 \%$ of this subset of cases to have a de novo CNV (Table 2). The highest de novo rate was for ASD (6.9\%), consistent with previous reports from 4.7 to $7.1 \%{ }^{43}$ For OCD, $1.8 \%$ had de novo CNVs, which is higher than the rate found in the general population $(0.9-1.4 \%),{ }^{43}$ but lower than $2.3 \%$ for OCD previously reported from a larger sample size. ${ }^{13}$

We observed deletions and duplications, other than those associated with known recurrent genomic disorders, in the same genes in different NDDs, including some in multiple cases (Fig. 2; Table 2, Supplementary Table 1D). Deletions impacted NRXN1 (in 9 males, 1 female) among ASD, ADHD, and SCZ cases (Fig. 2). Similarly, we found deletions impacting GNAL, LDLRAD4, SEH1L, DLGAP2, DCTN2, GRID2, and KIF5A among both ASD and ADHD cases (Fig. 2). ${ }^{10}$

Disruptive variants in gene-sets involved in multiple intracellular signaling pathways and DNA instability have been observed previously in ASD. ${ }^{23}$ Variants in gene pathways associated with DNA/"genomic instability" are increased in both ASD and SCZ. ${ }^{20}$ Consistent with these studies, we observed a 2.77-fold higher proportion of cases with NDD-CNVs among those with GIG-CNVs, than among those without.

This study had certain limitations. (i) Most cases, with the exception of SCZ, were recruited as children or adolescents on the basis of a specific diagnosis. ASD and ADHD have early onset, and many participants would not have reached the age for adolescent or adult-onset disorders, including OCD and SCZ. It is possible that individuals with early onset conditions will develop additional later-onset comorbidities. All SCZ cases in the current study were adults. (ii) Recruitment was by clinicians who focus on a single disorder. It is possible that some cases may have had other NDDs, which were not reported. For example, we had data on intellectual disability/IQ for the SCZ cohort and for some cases with other NDDs. We searched the genotype data for possible multiple ascertainment of any case and found no examples of subjects that were recruited through multiple disorders. We examined for non-primary phenotypes for specific cases with variants in NDD-relevant genes. (iii) Due to limitations of the technology, we studied CNVs only of a certain size $(>20 \mathrm{~kb})$ for the majority of samples where we did not have sequence data. Smaller CNVs and single nucleotide polymorphisms also contribute to the etiology of NDDs, ${ }^{8,35}$ but these would have been missed. A more sensitive technology such as genome sequencing would allow more comprehensive detection of all relevant variants. ${ }^{8,44}$ The dataset also needs to be analyzed iteratively as more data and better analysis tools become available. ${ }^{45}$

In summary, we highlighted clinically relevant CNVs found through microarray data for ASD, ADHD, OCD, and SCZ. We also demonstrated that identical CNVs or genes could potentially contribute to the etiology of multiple NDDs, consistent with previous reports, ${ }^{10,20,46,47}$ and providing a valuable resource for comparison in other studies.

\section{METHODS}

\section{Samples}

This project was a part of a multilateral collaborative project to investigate genetic etiology across four neurodevelopmental disorders: ADHD, ASD, OCD, and SCZ. This study was approved by the Research Ethics Board at The Hospital for Sick Children. A written informed consent was obtained from all participants or substitute decision makers. CNVs were detected on the same high-resolution microarray platform. The criteria for meeting a diagnosis of ASD, ADHD, OCD, or SCZ were detailed in our previous publications $8,10,13,23,24$ with a few modifications for ADHD (see Supplementary Information). Data from all OCD individuals and 139/435 (32\%) of the SCZ cohort had been 
previously published, ${ }^{11,13}$ but we included them here for comparative purposes (Supplementary Information). ADHD and ASD samples were not previously published. Additional supportive evidence for cross-disorder associations of selected CNVs came from our previously published schizophrenia cohorts ${ }^{11,24}$ and an additional ADHD cohort ${ }^{10}$; all were genotyped on the Affymetrix SNP 6.0 microarray (Table 3).

\section{Genotyping and detection of rare variants}

We extracted genomic DNA from saliva or blood and genotyped samples on the Affymetrix CytoScan HD platform. Quality control and ancestry assessment procedures were as discussed previously. ${ }^{25}$ Using PLINK v1.90b2, we found 1,995 (74.1\%) of cases to be of European ancestry (Supplementary Table 1A).

CNVs were identified as previously described. ${ }^{13,25}$ Briefly, four different algorithms were used to call high-confidence CNVs. These included the Affymetrix Chromosome Analysis Suite, iPattern, BioDiscovery Nexus, and Partek Genomics Suite. We defined a stringent set of variants of at least $20 \mathrm{~kb}$ wherein each was identified by at least two algorithms and spanned by at least five consecutive probes (Supplementary Table 1B). We defined rare CNVs as those present at no more than $0.1 \%$ frequency among 10,851 controls samples (detailed in Zarrei et al. ${ }^{25}$ ). We further restricted our list to those with more than $75 \%$ overlap with copy-number stable regions, according to our stringent CNV map of the human genome. ${ }^{2}$ We confirmed clinically relevant CNVs (Tables 1, 2, Supplementary Table 1C) (as defined below) using a SYBR ${ }^{\circledR}$ Green-based real-time quantitative PCR assays, TaqMan ${ }^{\circledR}$ copy number assays or whole genome sequencing data (if available). The genomic coordinates used are based on Human Genome Build GRCh37/hg19.

Prioritizing variants relevant to NDDs and the NDD gene list

To focus on CNVs relevant to NDDs, we first selected those variants coinciding with known recurrent genomic disorders, aneuploidies, and large $(>3 \mathrm{Mb})$ deletions and duplications. We also analyzed whether rare CNVs in our cases were similar to those in clinically relevant CNV databases at The Department of Paediatric Laboratory Medicine, The Hospital for Sick Children, comprising over 20,000 cases. We classified variants for their clinical impact according to American College of Medical Genetics guidelines. ${ }^{26}$ Our prioritized variants also included those impacting the coding sequences of genes with sufficient evidence for being clinically relevant to NDDs (Supplementary Information).

Cross-disorder gene discovery and genes in more than one case in a single disorder

We searched for genes that were impacted by CNVs of $20 \mathrm{~kb}$ to $3 \mathrm{Mb}$ in more than one case. Of these, we analyzed brain-expressed genes ${ }^{48}$ that were impacted by rare deletions and that are moderately to strongly constrained in the general population for LoF variants (as defined by a LoF probability of $\left.>0.45^{49} ; n=1,116\right)$. We also analyzed genes whose full transcript length was impacted by duplication.

Global burden test for protein-coding genes and IncRNAs

We performed a univariate analysis to test the global burden of variants impacting coding sequences of protein-coding genes and all exons of IncRNAs using a logistic regression model. We further tested a burden for brain-expressed protein-coding genes $(n=3,666)$ and IncRNAs $(n=1,070)$ to compare with those not expressed in the brain. Chromatin states from the Roadmap Epigenomics Consortium ${ }^{50}$ were used to identify brainexpressed genes (Supplementary Information). We defined controls as parents of cases in the regression analysis. We used sex and the first three principal components of population stratification calculated using PLINK as covariates. The model was also corrected for the total length of CNVs. Finally, we performed a multivariate analysis to investigate whether the burden signals were from the same sets of CNVs as in the univariate analysis (details in Supplementary Information). We considered $p<0.05$ as statistically significant. We also reported $0.05<p<0.1$ as nearly significant.

Genomic instability and fragile sites

Replication stress can lead to CNV formation, and fragile sites. A recent study using genome-wide $\mathrm{CNV}^{20}$ demonstrated a link between DNA/ genomic integrity and ASD and SCZ. However, using a larger sample size than the current study $(1,108$ ASD and 2,458 SCZ), they were unable to find pathways enriched in ASD versus SCZ and vice versa. Given smaller cohorts, we performed our analyses in a combined set of all four NDDs to achieve an acceptable statistical power. We first investigated CNVs impacting the coding sequences of genomic instability genes, looking for change in the proportion of cases with rare CNVs in these genes, compared with that of controls. The genomic instability genes comprised 958 protein coding genes identified from the AmiGO database ${ }^{36}$ by searching for the following terms: DNA repair, DNA replication, genome maintenance, DNA damage, and DNA integrity. We also tested for the overall number of rare CNVs and total length of rare CNVs. We then considered whether cases with perturbed genomic instability genes had a different burden of rare CNVs in NDD genes compared to that in cases with intact instability genes.

\section{Reporting summary}

Further information on experimental design is available in the Nature Research Reporting Summary linked to this paper.

\section{DATA AVAILIBILITY}

Relevant microarray data are deposited in the database of Genotypes and Phenotypes (https://www.ncbi.nlm.nih.gov/gap/; ID:phs001881.v1.p1). The relevant CNVs are available in dbVar (ID:nstd173).

Received: 6 May 2019; Accepted: 5 September 2019; Published online: 07 October 2019

\section{REFERENCES}

1. Lee, C. \& Scherer, S. W. The clinical context of copy number variation in the human genome. Expert Rev. Mol. Med. 12, e8 (2010).

2. Zarrei, M., MacDonald, J. R., Merico, D. \& Scherer, S. W. A copy number variation map of the human genome. Nat. Rev. Genet. 16, 172-183 (2015).

3. Scherer, S. W. \& Dawson, G. Risk factors for autism: translating genomic discoveries into diagnostics. Hum. Genet. 130, 123-148 (2011).

4. Smoller, J. W. et al. Psychiatric genetics and the structure of psychopathology. Mol. Psychiatry 24, 409-420 (2019).

5. Miller, D. T. et al. Consensus statement: chromosomal microarray is a first-tier clinical diagnostic test for individuals with developmental disabilities or congenital anomalies. Am. J. Hum. Genet. 86, 749-764 (2010).

6. Cook, E. H. Jr. \& Scherer, S. W. Copy-number variations associated with neuropsychiatric conditions. Nature 455, 919-923 (2008).

7. Pinto, D. et al. Functional impact of global rare copy number variation in autism spectrum disorders. Nature 466, 368-372 (2010).

8. Yuen, R. K. et al. Whole genome sequencing resource identifies 18 new candidate genes for autism spectrum disorder. Nat. Neurosci. 20, 602-611 (2017).

9. Marshall, C. R. et al. Structural variation of chromosomes in autism spectrum disorder. Am. J. Hum. Genet 82, 477-488 (2008).

10. Lionel, A. C. et al. Rare copy number variation discovery and cross-disorder comparisons identify risk genes for ADHD. Sci. Transl. Med. 3, 95ra75 (2011).

11. Lowther, C. et al. Impact of IQ on the diagnostic yield of chromosomal microarray in a community sample of adults with schizophrenia. Genome Med. 9, 105 (2017).

12. Marshall, C. R. et al. Contribution of copy number variants to schizophrenia from a genome-wide study of 41,321 subjects. Nat. Genet. 49, 27-35 (2017).

13. Gazzellone, M. J. et al. Uncovering obsessive-compulsive disorder risk genes in a pediatric cohort by high-resolution analysis of copy number variation. J. Neurodev. Disord. 8, 36 (2016).

14. McGrath, L. M. et al. Copy number variation in obsessive-compulsive disorder and tourette syndrome: a cross-disorder study. J. Am. Acad. Child Adolesc. Psychiatry 53, 910-919 (2014).

15. Faraone, S. V. \& Larsson, H. Genetics of attention deficit hyperactivity disorder. Mol. Psychiatry 24, 562-575 (2019).

16. Lowther, C. et al. Molecular characterization of NRXN1 deletions from 19,263 clinical microarray cases identifies exons important for neurodevelopmental disease expression. Genet Med. 19, 53-61 (2017).

17. Mercati, O. et al. CNTN6 mutations are risk factors for abnormal auditory sensory perception in autism spectrum disorders. Mol. Psychiatry 22, 625-633 (2017).

18. Cross-Disorder Group of the Psychiatric Genomics, C. Identification of risk loci with shared effects on five major psychiatric disorders: a genome-wide analysis. Lancet 381, 1371-1379 (2013). 
19. Gonzalez-Mantilla, A. J., Moreno-De-Luca, A., Ledbetter, D. H. \& Martin, C. L. A cross-disorder method to identify novel candidate genes for developmental brain disorders. JAMA Psychiatry 73, 275-283 (2016).

20. Kushima, I. et al. Comparative analyses of copy-number variation in autism spectrum disorder and schizophrenia reveal etiological overlap and biological insights. Cell Rep. 24, 2838-2856 (2018).

21. Niarchou, M. et al. Psychiatric disorders in children with $16 \mathrm{p} 11.2$ deletion and duplication. Transl. Psychiatry 9, 8 (2019).

22. Uddin, M. et al. A high-resolution copy-number variation resource for clinical and population genetics. Genet Med. 17, 747-752 (2015).

23. Prasad, A. et al. A discovery resource of rare copy number variations in individuals with autism spectrum disorder. G3 (Bethesda) 2, 1665-1685 (2012).

24. Costain, G. et al. Pathogenic rare copy number variants in community-based schizophrenia suggest a potential role for clinical microarrays. Hum. Mol. Genet. 22, 4485-4501 (2013).

25. Zarrei, M. et al. De novo and rare inherited copy-number variations in the hemiplegic form of cerebral palsy. Genet. Med. 20, 172-180 (2018).

26. Kearney, H. M. et al. American College of Medical Genetics standards and guidelines for interpretation and reporting of postnatal constitutional copy number variants. Genet. Med. 13, 680-685 (2011).

27. Yuen, R. K. et al. Whole-genome sequencing of quartet families with autism spectrum disorder. Nat. Med. 21, 185-191 (2015).

28. Jiang, Y. H. et al. Detection of clinically relevant genetic variants in autism spectrum disorder by whole-genome sequencing. Am. J. Hum. Genet. 93, 249-263 (2013).

29. van Rijn, S., de Sonneville, L. \& Swaab, H. The nature of social cognitive deficits in children and adults with Klinefelter syndrome (47,XXY). Genes Brain Behav. 17, e12465 (2018).

30. Lionel, A. C. et al. Disruption of the ASTN2/TRIM32 locus at $9 q 33.1$ is a risk factor in males for autism spectrum disorders, ADHD and other neurodevelopmental phenotypes. Hum. Mol. Genet. 23, 2752-2768 (2014).

31. Duong, L. T. et al. Two rare deletions upstream of the NRXN1 gene ( $2 p 16.3)$ affecting the non-coding mRNA AK127244 segregate with diverse psychopathological phenotypes in a family. Eur. J. Med. Genet. 58, 650-653 (2015).

32. Rizzo, A. et al. The noncoding RNA AK127244 in 2p16.3 locus: a new susceptibility region for neuropsychiatric disorders. Am. J. Med. Genet. B Neuropsychiatr. Genet. 177, 557-562 (2018).

33. Nguyen, T. \& Di Giovanni, S. NFAT signaling in neural development and axon growth. Int J. Dev. Neurosci. 26, 141-145 (2008).

34. Noor, A. et al. Disruption at the PTCHD1 Locus on Xp22.11 in Autism spectrum disorder and intellectual disability. Sci. Transl. Med. 2, 49ra68 (2010).

35. Yuen, R. K. et al. Genome-wide characteristics of de novo mutations in autism. NPJ Genom. Med. 1, 160271-1602710 (2016).

36. Carbon, S. et al. AmiGO: online access to ontology and annotation data. Bioinformatics 25, 288-289 (2009).

37. Nikkila, J. et al. Heterozygous mutations in PALB2 cause DNA replication and damage response defects. Nat. Commun. 4, 2578 (2013).

38. Wei, P. C. et al. Long neural genes harbor recurrent DNA break clusters in neural stem/progenitor cells. Cell 164, 644-655 (2016).

39. Mauger, C. et al. Executive functions in children and adolescents with turner syndrome: a systematic review and meta-analysis. Neuropsychol. Rev. 28, 188-215 (2018).

40. Molloy, C. A. et al. Differences in the clinical presentation of Trisomy 21 with and without autism. J. Intellect. Disabil. Res. 53, 143-151 (2009).

41. Kendall, K. M. et al. Cognitive performance among carriers of pathogenic copy number variants: analysis of 152,000 UK biobank subjects. Biol. Psychiatry 82, 103-110 (2017).

42. Lowther, C., Costain, G., Baribeau, D. A. \& Bassett, A. S. Genomic disorders in psychiatry-what does the clinician need to know? Curr. Psychiatry Rep. 19, 82 (2017).

43. Oskoui, M. et al. Clinically relevant copy number variations detected in cerebral palsy. Nat. Commun. 6, 7949 (2015).

44. Trost, B. et al. A comprehensive workflow for read depth-based identification of copy-number variation from whole-genome sequence data. Am. J. Hum. Genet. 102, 142-155 (2018).

45. Costain, G. et al. Periodic reanalysis of whole-genome sequencing data enhances the diagnostic advantage over standard clinical genetic testing. Eur. J. Hum. Genet. 26, 740-744 (2018).

46. Diaz-Beltran, L. et al. Cross-disorder comparative analysis of comorbid conditions reveals novel autism candidate genes. BMC Genomics 18, 315 (2017).

47. Moreno-De-Luca, D., Moreno-De-Luca, A., Cubells, J. F. \& Sanders, S. J. Crossdisorder comparison of four neuropsychiatric CNV loci. Curr. Genet. Med. Rep. 2, 151-161 (2014).

48. Lukk, M. et al. A global map of human gene expression. Nat. Biotechnol. 28, 322-324 (2010).

49. Lek, M. et al. Analysis of protein-coding genetic variation in 60,706 humans. Nature 536, 285-291 (2016)
50. Roadmap Epigenomics, C. et al. Integrative analysis of 111 reference human epigenomes. Nature 518, 317-330 (2015).

\section{ACKNOWLEDGEMENTS}

We thank Christina Chrysler, Lili Senman, Carolyn Russell, Rebecca Baatjes, Ann Thompson, Mary Ann George, Berivan Baskin, Jessica Rickaby, Susan D. Fragiadakis, Leanne Ristic, Alana laboni, Mathew J. Gazzellone, S-M Shaheen, Rageen Rajendram, Wilson Sung, and Melissa Hudson for technical assistance and helpful discussions. The authors also thank the patients, families, and referring clinicians. We also thank Julie Coste for assistance with data management of the OCD samples, Reva Schachter and Marlena Colasanto for assistance in recruitment and phenotyping of OCD participants. This study was supported by grants from Genome Canada, Canada Foundation for Innovation, Canadian Institute for Advanced Research, Government of Ontario, Canadian Institutes of Health Research, The Hospital for Sick Children Foundation, Ontario Brain Institute $(\mathrm{OBI}) /$ The Province of Ontario Neurodevelopmental Disorders Network (POND), Autism Speaks, and University of Toronto McLaughlin Centre. The MSSNG-WGS sequencing and open science platform is supported by Autism Speaks and partners. This research is also supported by MOP209699 and MOP-192190 grants from Canadian Institutes of Health Research and IDSI I-02 from OBI. G.L.H., D.R.R., and P.D.A. were supported by R01MH085321 grant from National Institute of Mental Health. G.L.H. and P.D.A. were supported by R01MH101493 grant also from National Institute of Mental Health. D.R.R. was further supported by Children's Foundation of Michigan, Lycaki-Young funds State of Michigan, Miriam Hamburger Endowment and Paul and Anita Strauss Endowment. P. D.A. holds the Alberta Innovates Translational Health Chair in Child and Youth Mental Health. S.W.S. holds the GlaxoSmithKline-CIHR Chair in Genome Sciences at The Hospital for Sick Children and University of Toronto. The following section acknowledge the control samples used in the current study. (i) Funding support for the Study of Addiction: Genetics and Environment (SAGE) was provided through the NIH Genes, Environment and Health Initiative [GEI] (U01 HG004422). SAGE is one of the genome-wide association studies funded as part of the Gene Environment Association Studies (GENEVA) under GEl. Assistance with phenotype harmonization and genotype cleaning, as well as with general study coordination, was provided by the GENEVA Coordinating Center (U01 HG004446). Assistance with data cleaning was provided by the National Center for Biotechnology Information. Support for collection of datasets and samples was provided by the Collaborative Study on the Genetics of Alcoholism (COGA; U10 AA008401), the Collaborative Genetic Study of Nicotine Dependence (COGEND; P01 CA089392), and the Family Study of Cocaine Dependence (FSCD; R01 DA013423). Funding support for genotyping, which was performed at the Johns Hopkins University Center for Inherited Disease Research, was provided by the NIH GEI (U01HG004438), the National Institute on Alcohol Abuse and Alcoholism, the National Institute on Drug Abuse, and the NIH contract "High throughput genotyping for studying the genetic contributions to human disease" (HHSN268200782096C). The datasets used for the analyses described in this manuscript were obtained from dbGaP at http://www.ncbi.nlm.nih.gov/projects/ gap/cgi- bin/study.cgi?study_id = phs000092.v1.p1 through dbGaP accession number phs000092.v1.p1. (ii) The authors acknowledge the contribution of data from Genetic Architecture of Smoking and Smoking Cessation accessed through dbGaP. Funding support for genotyping, which was performed at the Center for Inherited Disease Research (CIDR), was provided by $1 \times 01$ HG005274-01. CIDR is fully funded through a federal contract from the National Institutes of Health to The Johns Hopkins University, contract number HHSN268200782096C. Assistance with genotype cleaning, as well as with general study coordination, was provided by the Gene Environment Association Studies (GENEVA) Coordinating Center (U01 HG004446). Funding support for collection of datasets and samples was provided by the Collaborative Genetic Study of Nicotine Dependence (COGEND; P01 CA089392) and the University of Wisconsin Transdisciplinary Tobacco Use Research Center (P50 DA019706, P50 CA084724). The datasets used for the analyses described in this manuscript were obtained from dbGaP at https://www.ncbi.nlm.nih.gov/projects/ gap/cgi-bin/study.cgi?study_id=phs000404.v1.p1 through dbGaP accession number phs000404.v1.p1. The dataset(s) used for the analyses described in this manuscript were obtained from the NEI Refractive Error Collaboration (NEIREC). Funding support for NEIREC was provided by the National Eye Institute. We would like to thank NEIREC participants and the NEIREC Research Group for their valuable contribution to this research. The datasets used for the analyses described in this manuscript were obtained from dbGaP at https://www.ncbi.nlm.nih.gov/projects/gap/cgi-bin/study. cgi?study_id=phs000303.v1.p1 through dbGaP accession number phs000303.v1.p1. (iii) Funding support for the "CIDR Visceral Adiposity Study" was provided through the Division of Aging Biology and the Division of Geriatrics and Clinical Gerontology, NIA. The CIDR Visceral Adiposity Study includes a genome-wide association study funded as part of the Division of Aging Biology and the Division of Geriatrics and Clinical Gerontology, NIA. Assistance with phenotype harmonization and genotype cleaning, as well as with general study coordination, was provided by Heath $A B C$ Study Investigators. The datasets used for the analyses described in this manuscript 
were obtained from dbGaP at https://www.ncbi.nlm.nih.gov/projects/gap/cgi-bin/ study.cgi?study_id=phs000169.v1.p1 through dbGaP accession number phs000169. v1.p1.

\section{AUTHOR CONTRIBUTIONS}

M.Z. and S.W.S. conceived and designed the experiments. M.Z., C.L.B., W.E., E.J.Y., E.J. H., J.W., J.R.M., K.R., G.P., M.F., A.J.C., B.D.M., S.W., B.T., M.W., and D.M processed and analysed the microarray data. S.L. designed and performed experiments for variant characterization and validation. C.L., T.W., K.S., and X.W. performed the CytoScan HD microarray laboratory experiments. B.K. and J.L.W. helped perform different components of analysis and sample collection. M.Z., E.J.Y., E.J.H., G.C., M.R., R.K.C.U., J.A.B., J.A.S.V., R.V.P., C.R.M., R.F.W., and D.J.S. helped perform different components of analysis and data interpretations. M.W., C.C., L.Z., M.E., J.F., B.A.F., M.T.C., P.Z., X.L., R.N., G.L.H., D.R.R., S.G., R.W., T.G., M.S., I.D., M.W.S., P.D.A., A.S.B., J.C., R.S., and E.A. diagnosed, examined and recruited the participants. M.Z. and S.W.S. wrote the manuscript.

\section{COMPETING INTERESTS}

S.W.S. serves on the Scientific Advisory Committees of Population Bio and Deep Genomics; intellectual property originating from his research and held at the Hospital for Sick Children is licensed to Lineagen, and separately Athena Diagnostics. D.M. is a full-time employee of Deep Genomics and is entitled to a stock option. R.J.S., P.D.A., and J.C. consult for Highland Therapeutics. Intellectual property from ADHD research at the Hospital for Sick Children is licensed to Ehave and the National Research Council of Canada. Other authors declare no competing interests for the data and interpretation presented in this study. R.J.S., P.D.A., and J.C. consults for Highland Therapeutics. Intellectual property from their research at the Hospital for Sick Children is licensed to Ehave and the National Research Council. D.M. is a full-time employee of Deep Genomics and is entitled to stock options. S.W.S. is on the Scientific Advisory Committees of Population Bio and Deep Genomics; intellectual property from his research held at the Hospital for Sick Children is licensed to Athena
Diagnostics, and separately to Lineagen. These relationships did not influence data interpretation or presentation during this study, but are disclosed for potential future consideration.

\section{ADDITIONAL INFORMATION}

Supplementary information is available for this paper at https://doi.org/10.1038/ s41525-019-0098-3.

Correspondence and requests for materials should be addressed to S.W.S

Reprints and permission information is available at http://www.nature.com/ reprints

Publisher's note Springer Nature remains neutral with regard to jurisdictional claims in published maps and institutional affiliations.

(i) Open Access This article is licensed under a Creative Common Attribution 4.0 International License, which permits use, sharing, adaptation, distribution and reproduction in any medium or format, as long as you give appropriate credit to the original author(s) and the source, provide a link to the Creative Commons license, and indicate if changes were made. The images or other third party material in this article are included in the article's Creative Commons license, unless indicated otherwise in a credit line to the material. If material is not included in the article's Creative Commons license and your intended use is not permitted by statutory regulation or exceeds the permitted use, you will need to obtain permission directly from the copyright holder. To view a copy of this license, visit http://creativecommons. org/licenses/by/4.0/.

(c) The Author(s) 2019 